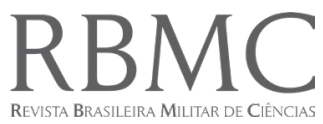

ISSN 2447-9071

doi $10.36414 /$ rbmc.v5i12.9
Contato para correspondência:

Clayson Moura Gomes

E-mail:

claysonmoura10@gmail.com

Conflito de interesse: Não

Financiamento: Recursos próprios

Recebido: 24/06/2019

Aprovado: 13/08/2019

\section{Avaliação de variáveis analíticas na doença renal crônica em pacientes atendidos em laboratórios de Goiânia - Goiás}

\author{
Some of variable rate analytical in chronic kidney disease \\ patients treated in Goiânia laboratories - Goiás
}

Ana Beatriz Sobral Peixoto', Kassya Polliana Sousa Santos Jaime², José Rodrigues de Melo Neto ${ }^{3}$, Sérgio Henrique Nascente Costa ${ }^{4}$, Karlla Greick Batista Dias Penna ${ }^{5}$, Clayson Moura Gomes ${ }^{6}$

${ }^{1}$ Biomédica, Pontifícia Universidade Católica de Goiás, ${ }^{2}$ Biomédica, Pontifícia Universidade Católica de Goiás, ${ }^{3}$ Biomédico, Mestre em Assistência e Avaliação de Saúde pela UFG, ${ }^{4}$ Biomédico, Doutor em Ciências da Saúde, Professor da PUC Goiás, Professor da UFG, Coordenador Geral Acadêmico da Faculdade da Polícia Militar, ${ }^{5}$ Biomédica, Doutora em Biologia Celular e Molecular pela UFG, Coordenadora do curso de Biomedicina da PUC Goiás, ${ }^{6}$ Biomédico, Doutor em Medicina Tropical e Saúde Pública pela UFG, Professor da PUC Goiás, Coordenador do curso de Biomedicina da Faculdade da Polícia Militar.

\section{Resumo}

A doença renal crônica (DRC) é considerada uma das principais causas de óbito elou incapacidade no mundo, porém são poucas as publicações em Goiás. A proposta do presente estudo foi comparar algumas variáveis laboratoriais associadas à DRC e correlacionar esses dados encontrados com as variáveis, idade e sexo. Estudo transversal retrospectivo de levantamento de dados de 210 pacientes do Laboratório Clínico da PUC Goiás (LAC - PUC GO) e do Hospital do Policial Militar de Goiás (HPM GO) no período de junho de 2013 a junho de 2014. A análise de comparação das médias de variáveis entre os grupos foi realizada pelo Teste t-Student e a análise de correlação pelo Teste de Spearman e Pearson, conforme a normalidade das variáveis. Nos pacientes masculinos do grupo teste, encontrouse correlação linear significativa entre hemoglobina e a TFG $(r=0,37$-regular, $p<0,0001)$ e com a idade $(r=0,3$-regular, $p=0,004)$. Quanto àvariável idade observou-se que houve correlação significativa com os valores de creatinina ( $r=0,47$ - regular, $p=0,02)$ nos pacientes do sexo feminino do grupo teste $e$ com a TFG $(r=0,6$ - regular, $p<0,0001)$ nos pacientes do sexo masculino do grupo teste. As variáveis avaliadas apresentaram correlação com a doença renal crônica, sendo que as de maior significância foram idade do paciente, valor de hemoglobina e TFG.

Palavras-Chave: Doença Renal Crônica, Anemia, TFG, Obesidade.

\begin{abstract}
Chronic kidney disease (CKD) is considered a major cause of death and/or disability worldwide. It is characterized by progressive and irreversible loss of function inan increasing number of nephrons, which gradually decrease the kidney's functions. The anemia resulting from chronic kidney disease may be due to deficiency in the production of erythropoietin, as well as iron deficiency caused by proinflammatory cytokines. Cross-sectional study and retrospective data collection from 210 patients in the Clinical Laboratory of PUC Goiás (LAC-PUCGO) and the Hospital Police Officer of Goiás (GOHPM) from June 2013 to June 2014. The analysis comparing the means of variables between groups was performed by Student's t-test and correlation analysis by Spearman test and Pearson, as the normality of variables. In male test group patients, there was a significant linear correlation between hemoglobin and GlomerularFiltration Rate (GFR) $(r=0.37$-regular, $p<0.0001)$ and age $(r=0.3$-regulate, $p=0.004)$. As for the variable age, it was observed a significant correlation with creatinine values ( $r=0.47$ - regular, $p=0.02)$ in female patients on the test group and $G F R(r=0.6$ - regular, $p<0.0001)$ in malepatients on the test group. The variables evaluated were correlated with CKD and the most significant ones were the patient's age, hemoglobin level and GFR.
\end{abstract}

Palavras-Chave: Chronic Kidney Disease, Anemia, GFR, Obesity. 


\section{Introdução}

Os rins são órgãos importantes para a homeostase do organismo humano, atuando na filtração sanguínea, reabsorção de elementos importantes, excreção de toxinas e funções endócrinas ${ }^{1,2}$. Sendo assim, a Doença Renal Crônica (DRC) é considerada uma das principais causas de óbito e/ou incapacidade no mundo'. É caracterizada por perda progressiva e irreversível da função e do número dos néfrons, que diminuem gradativamente a função dos rins. Laboratorialmente, só é possível observar alteração nos níveis séricos após a perda de 50 a $60 \%$ da função dos néfrons ${ }^{3,4}$. A DRC pode ser decorrente de distúrbios nos vasos sanguíneos, glomérulos, túbulos, interstício renal e trato urinário inferior ${ }^{5}$.

A DRC é um estado de inflamação em que pode ser observada a presença de alguns marcadores inflamatórios aumentados, como a proteína $\mathrm{C}$ reativa, haptoglobina, a1glicoproteína-ácida e citocinas pró-inflamatórias, como interleucina (IL) 1 e 6 e fator de necrose tumoral (TNF) alfa. Esses marcadores inflamatórios interferem na síntese e função da eritropoietina, além de prejudicar a mobilização dos estoques e a absorção intestinal de ferro ${ }^{6,7}$.

A deficiência na produção de eritropoietina, que é regulada pela concentração de hemoglobina, é um dos fatores que desencadeia a anemia decorrente da DRC. Assim, quando ocorre uma diminuição da oxigenação celular, as células intersticiais do córtex renal são estimuladas a produzir o Fator Hypoxiainducible alfa (HIF-a) que é um estimulador da produção de eritropoietina ${ }^{8}$.

A inflamação crônica contribui para a produção hepática de hepcidina, que é um peptídeo responsável por inibir a mobilização de ferro dos seus estoques, tem uma atuação tanto nos enterócitos inibindo a absorção de ferro, quanto na liberação do ferro pelos macrófagos e enterócitos ${ }^{9}$. A hepcidina liga-se com a ferroportina degradando-a e impedindo a liberação de ferro pelos macrófagos ${ }^{8,10}$, levando a um quadro de anemia.

Na DRC avalia-se o grau de lesão pela taxa de filtração glomerular (TFG) que é a capacidade dos rins de filtrar substâncias provenientes do sangue ${ }^{11}$. Essa avaliação é realizada através da dosagem de creatinina sérica e/ou pela depuração de creatinina na urina de 24 horas, isso é possível porque a creatinina é uma substância produzida pelo organismo, completamente excretada pelos rins e não é reabsorvida ${ }^{5}$, sendo que esta avaliação varia de acordo com a idade, sexo e massa muscular. Na DRC, a secreção da creatinina diretamente pelos túbulos contorcidos proximais aumenta cerca de 15 a 20\%, caracterizando um interferente nos cálculos da TFG. Um resultado de TFG de $70 \mathrm{~mL} /$ $\mathrm{min} / 1,73 \mathrm{~m}^{2}$ em homens e $50 \mathrm{~mL} / \mathrm{min} / 1,73 \mathrm{~m}^{2}$ em mulheres corresponde a perda de aproximadamente $50 \%$ da função renal ${ }^{8}$.
Os principais fatores considerados de risco para o desenvolvimento da DRC são obesidade, diabetes mellitus (DM), hipertensão arterial (HA) ea idade ${ }^{12}$. Isso porque a obesidadeécapaz de aumentar a dimensão dos glomérulos, além de favorecer a presença de anormalidades na função glomerular e de glomeruloesclerose segmentar e focal (GESF) ${ }^{13}$. A perda de peso auxilia na diminuição de albumina na urina e a excessiva filtração glomerular em pacientes com obesidade mórbida ${ }^{3}$. Além disso, a obesidade está presente em cerca de $90 \%$ dos pacientes com diabetes mellitus tipo 2, e 65 a 75\% dos pacientes com hipertensão arterial primária ${ }^{13}$.

Considerando que a prevenção de doenças ocupacionais numa população composta por Policiais Militares ativos, inativos e dependentes seria de interesse para evitar os riscos profissionais peculiares no seu processo de trabalho e considerando ainda, que na DRC alguns pacientes são assintomáticos e que a melhor estratégia é a prevenção primária, o objetivo deste estudo foi comparar algumas variáveis laboratoriais associadas à DRC entre grupos de pacientes que apresentavam ou não alteração renal, correlacionando os dados encontrados às variáveis sexo e idade.

\section{Métodos}

Estudo do tipo transversal de levantamento de dados de pacientes do Laboratório Clínico da PUC Goiás (LAC - PUC Goiás) e do Hospital do Policial Militar de Goiás (HPM) no período de junho de 2013 a junho de 2014, aprovado pelo Comitêde Ética em Pesquisa da PUC-Goiás sob o parecer de número 235.376/2013.

Um total de 210 pacientes foram incluídos neste estudo, o que representa cerca de $20 \%$ dos pacientes atendidos em ambos os laboratórios que realizaram dosagem de creatinina sérica. Os critérios de inclusão foram idadeacima de 40 anos ea realização de dosagens de creatinina sérica e hemograma. O grupo controle foi composto por 100 pacientes sem doença renal, enquanto que o grupo teste foi constituído por 110 pacientes portadores de doença renal. A alteração renal foi definida de acordo com os valores séricos de creatinina, acima de $1,3 \mathrm{mg} / \mathrm{dL}$ para homense $1,2 \mathrm{mg} / \mathrm{dL}$ para mulheres e da TFG para sexo e idade. Foi utilizada a fórmula de Cockroft-Gault [(140-idade) xpeso]/(creatinina séricax 72$)]$ para homens, e para mulheres multiplica ovalor por 0,85 para o cálculo daTFG14. As dosagens decreatinina sérica foram realizadas no equipamento automatizado Selectra Pro XL, utilizando o kit Creatinine Jaffe da marca ELITech ${ }^{\circledast}$ com a metodologia cinética colorimétrica no LAC - PUC Goiás e no equipamento $A 15$ utilizando kits BioSystems ${ }^{\circledR}$ no HPM.

A DRC foi classificada, segundo a Kidney Disease Outcome Quality Initiative (KDOQI), em 5 estágios: estágio 1 (TFG $\left.\geq 90 \mathrm{~mL} / \mathrm{min} / 1,73 \mathrm{~m}^{2}\right)$, estágio $2\left(\mathrm{TFG} 60 \mathrm{~mL} / \mathrm{min} / 1,73 \mathrm{~m}^{2}\right.$ a $89 \mathrm{~mL} / \mathrm{min} / 1,73 \mathrm{~m}^{2}$ ); estágio $3\left(\mathrm{TFG} 30 \mathrm{~mL} / \mathrm{min} / 1,73 \mathrm{~m}^{2}\right.$ a $59 \mathrm{~mL} / \mathrm{min} / 1,73 \mathrm{~m}^{2}$ ); estágio 4 (TFG $15 \mathrm{~mL} / \mathrm{min} / 1,73 \mathrm{~m}^{2}$ a $29 \mathrm{~mL} /$ $\mathrm{min} / 1,73 \mathrm{~m}^{2}$ ) e estágio $5\left(\mathrm{TFG}<15 \mathrm{~mL} / \mathrm{min} / 1,73 \mathrm{~m}^{2}\right)^{1}$. 
Por meio do hemograma automatizado, realizado em equipamento XE-2100 da Sysmex ${ }^{\circledR}$ no LAC-PUC Goiás, e pelo equipamento $A B X$ Pentra 60 no HPM, foi avaliado a presença de anemia de acordo com os níveis séricos de hemoglobina abaixo de $13 \mathrm{mg} / \mathrm{dL}$ em homens e $12 \mathrm{mg} / \mathrm{dL}$ em mulheres, segundo a OMS5,15. Foram coletados ainda os valores de peso e altura de todos os pacientes para cálculo do Índice de Massa Corporal (IMC) e a relação de medicamentos em uso.

Para o cálculo das médias, medianas e desvio padrão foi utilizado o software PAST versão 3.11. A análise de comparação das médias de variáveis entre os grupos foi realizada pelo Teste $t$ Student. Para a análise de correlação foi utilizado o Teste de Spearmane Pearson, conforme a normalidade das variáveis. Os valores de $p<0,05$ foram considerados estatisticamente significantes.

\section{Resultados}

Do total de 210 pacientes incluídos neste estudo, 34,8\% $(73 / 210)$ eram do sexo feminino e $65,2 \%$ (137/210) do sexo masculino. $O$ grupo controle representado por 100 pacientes apresentou uma mediana de idade de 60 anos, sendo o intervalo de 40 a 83 anos. $O$ grupo teste com um total de 110 pacientes apresentou a mediana de idade de 70 anos com intervalo de 42 a 88 anos. Dentre as 24 mulheres do grupo teste $58,3 \%(14 / 24)$ estavam na faixa etária maior que 70 anos e 52,3\% (45/86) dos homens estavam na mesma faixa etária.

Foram avaliados alguns fatores de risco que estão associados à progressão para a perda da função renal tais como HA, DM, idade acima de 60 anos e sobrepeso ou obesidade $\left(\right.$ IMC $\left.>25 \mathrm{~kg} / \mathrm{m}^{2}\right) 5$. Neste estudo 44,5\% (49/110) dos pacientes do grupo teste foram classificados como hipertensos baseados nos medicamentos em uso e 4,5\% (5/110) como diabéticos, sendo que 13,6\% (15/110) apresentaram diabetes e hipertensão simultaneamente. Do total de pacientes do grupo teste $91,8 \%$ (101/110) apresentaram idade acima de 60 anos, considerados idosos e 67,3\% (74/110) com o IMC acima da normalidade foram classificados como obesos ou sobrepeso e são mostrados na figura 1.

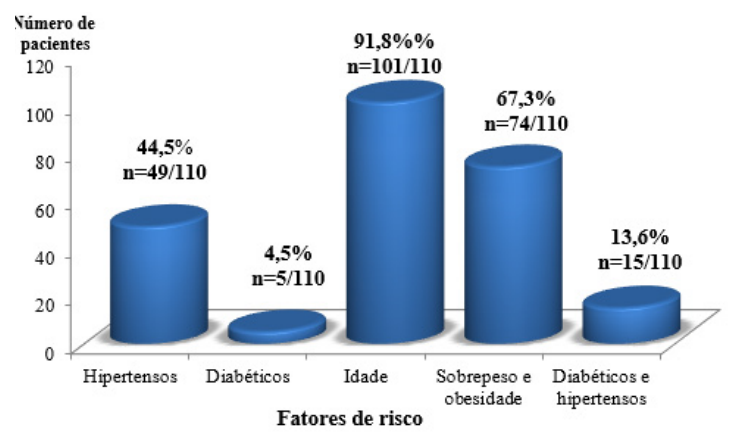

Figura 1: Fatores de risco para o desenvolvimento de DRC. Legenda: DM - diabetes mellitus; HAS - hipertensão arterial sistêmica; DRC - doença renal crônica.
A tabela 1 mostra o perfil da amostra estudada em associação com a presença de anemia. A anemia esteve presente em 31,8\% (35/110) dos pacientes do grupo teste em relação a $7 \%(7 / 100)$ do grupo controle. Dentre os 35 pacientes com anemia no grupo teste, $34,3 \%$ (12/35) eram do sexo feminino e $65,7 \%$ (23/35) eram do sexo masculino e ambos estavam na faixa etária acima de 60 anos. No grupo controle 57,1\% (04/07) dos pacientes do sexo masculino apresentaram anemia em relação a 42,8\% (03/07) do sexo feminino, distribuídos em outras faixas etárias.

Tabela 1. Distribuição dos pacientes do grupo controle e do grupo teste de acordo com a faixa etária, sexo e presença de anemia, Goiânia, 2014.

\begin{tabular}{|c|c|c|c|c|c|}
\hline & & Mediana & $\begin{array}{l}\text { Idade } \\
\text { (anos) }\end{array}$ & $\mathbf{N}$ & $\begin{array}{c}\text { Com } \\
\text { anemia }\end{array}$ \\
\hline \multirow{10}{*}{ Grupo Controle } & & & $40-49$ & 14 & 1 \\
\hline & & & $50-59$ & 7 & 0 \\
\hline & Masculinos & 60 & & & \\
\hline & & & $60-69$ & 20 & 1 \\
\hline & & & $>70$ & 10 & 2 \\
\hline & & & $40-49$ & 15 & 0 \\
\hline & & & $50-59$ & 10 & 2 \\
\hline & Femininos & 58 & & & \\
\hline & & & $60-69$ & 11 & 0 \\
\hline & & & $>70$ & 13 & 1 \\
\hline \multirow{10}{*}{ Grupo Teste } & & & $40-49$ & 2 & 0 \\
\hline & & & $50-59$ & 4 & 0 \\
\hline & Masculinos & 70 & & & \\
\hline & & & $60-69$ & 35 & 7 \\
\hline & & & $>70$ & 45 & 16 \\
\hline & & & $40-49$ & 1 & 0 \\
\hline & & & $50-59$ & 2 & 0 \\
\hline & Femininos & 71 & & & \\
\hline & & & $60-69$ & 7 & 4 \\
\hline & & & $>70$ & 14 & 8 \\
\hline Total & & & & 210 & 42 \\
\hline
\end{tabular}

Em relação aos dados apresentados na tabela 2, do total de 24 pacientes do sexo feminino do grupo teste, 10/24 foram classificadas como estágio 3 da DRC sendo que 5/10 apresentaram anemia. Um total de 13/24 mulheres foram classificadas como estágio 4 da DRC e destas, 7/13 apresentaram anemia. $1 / 24$ paciente foi classificada como estágio 5 da DRC e não apresentou anemia. No que se refere ao sexo masculino, dos 86 pacientes do grupo teste, $57 / 86$ foram classificados como 
estágio 3 da DRC, sendo que 18/57 apresentaram anemia. 9/86 pacientes foram classificados como estágio 4 da DRC e destes, 4/9 apresentaram anemia, e 1/86 foi classificado como estágio 5 da DRC e apresentou anemia.

Tabela 2. Distribuição dos pacientes do sexo feminino e masculino, segundo estágios da DRC, Goiânia, 2014.

\begin{tabular}{lcccc}
\hline & Sexo Feminino $(\mathbf{n}=\mathbf{2 4})$ & Sexo Masculino $(\mathbf{n}=\mathbf{8 6})$ \\
Estágios & $\mathbf{N}$ & Anemia & N & Anemia \\
& 10 & 5 & 57 & 18 \\
\hline Estágio 3 & 13 & 7 & 9 & 4 \\
Estágio 4 & 1 & 0 & 1 & 1 \\
\hline Estágio 5 & & & & \\
\hline
\end{tabular}

A tabela 3 demonstra os resultados das análises da correlação entre as variáveis dos pacientes dos grupos controle e teste distribuído por sexo. Na análise de correlação entre os pacientes com hemoglobina abaixo do normal e a idade, foi possível observar uma correlação linear significativa nos pacientes femininos do grupo teste $(r=0,9$ - muito forte, $p<0,0001)$. Entre os pacientes masculinos do grupo teste, encontrou-se correlação linear significativa entre hemoglobina e a TFG $(r=0,37$ - regular, $p<0,0001)$ e com a idade $(r=0,3$ - regular, $p=0,004)$.

Os valores do IMC correlacionaram-se com a TFG ( $r=0,5$ - regular, $p=0,01)$ nos pacientes do sexo feminino do grupo teste; enquanto que nos pacientes do sexo masculino do grupo teste, houve correlação com os valores de hemoglobina $(r=0,26$ - fraca, $p=0,02), \operatorname{TFG}(r=0,54$ - regular, $p<0,0001)$, e com a idade $(r=0,36$ - regular, $p=0,001)$.

Tabela 3. Correlação entre as variáveis dos pacientes do grupo controle e teste distribuídos por sexo, Goiânia, 2014.

\begin{tabular}{ccccccccc}
\hline \multirow{2}{*}{ Grupos } & Variáveis & \multicolumn{2}{c}{ Hemoglobina } & \multicolumn{2}{c}{ IMC } & \multicolumn{2}{c}{ Idade } \\
\cline { 3 - 8 } & & $\boldsymbol{r}$ & $\boldsymbol{p}$ & $\boldsymbol{r}$ & $\boldsymbol{p}$ & $\boldsymbol{r}$ & $\boldsymbol{p}$ \\
\hline $\begin{array}{c}\text { Grupo } \\
\text { Teste }\end{array}$ & TFG & 0,1 & 0,65 & $0,5^{*}$ & $0,01^{*}$ & $-0,34$ & 0,09 \\
$\begin{array}{c}\text { Creatinina } \\
\text { Idade }\end{array}$ & 0,00021 & 0,99 & - & - & $-0,47^{*}$ & $0,02^{*}$ \\
& Hemoglobina & - & $-0,9^{*}$ & $<0,0001^{*}$ & $-0,17$ & 0,42 & - & - \\
\hline
\end{tabular}

\begin{tabular}{|c|c|c|c|c|c|c|c|}
\hline \multirow{4}{*}{$\begin{array}{c}\text { Grupo } \\
\text { Controle } \\
\text { Feminino }\end{array}$} & Creatinina & 0,77 & 0,19 & - & - & 0,28 & 0,06 \\
\hline & TFG & 0,15 & 0,29 & 0,26 & 0,06 & $-0,66^{*}$ & $\begin{array}{c}< \\
0,0001^{*}\end{array}$ \\
\hline & Idade & $-0,2$ & 0,15 & 0,13 & 0,39 & - & - \\
\hline & Hemoglobina & - & - & $0,49^{*}$ & $<0,0001^{*}$ & - & - \\
\hline \multirow{4}{*}{$\begin{array}{c}\text { Total } \\
\text { Feminino }\end{array}$} & Creatinina & $-0,32$ & 0,006 & - & - & 0,17 & 0,15 \\
\hline & TFG & 0,48 & $<0,0001$ & 0,46 & $<0,0001$ & $-0,68$ & $<0,0001$ \\
\hline & Idade & $-0,9$ & $<0,0001$ & $-0,14$ & 0,22 & - & - \\
\hline & Hemoglobina & - & - & 0,42 & $<0,0001$ & - & - \\
\hline \multirow{4}{*}{$\begin{array}{c}\text { Grupo } \\
\text { Teste } \\
\text { Mascu- } \\
\text { lino }\end{array}$} & Creatinina & $-0,18$ & 0,08 & - & - & $-0,01$ & 0,91 \\
\hline & TFG & $0,37^{*}$ & $<0,0001^{*}$ & $0,54^{*}$ & $<0,0001^{*}$ & $-0,6^{*}$ & $\begin{array}{c}< \\
0,0001^{*}\end{array}$ \\
\hline & Idade & $-0,3^{*}$ & $0,004^{*}$ & $-0,36^{*}$ & $0,001^{*}$ & - & - \\
\hline & Hemoglobina & - & - & $0,26^{*}$ & $0,02^{*}$ & - & - \\
\hline \multirow{4}{*}{$\begin{array}{c}\text { Grupo } \\
\text { Controle } \\
\text { Mascu- } \\
\text { lino }\end{array}$} & Creatinina & 0,19 & 0,17 & - & - & $-0,2$ & 0,14 \\
\hline & TFG & 0,2 & 0,16 & $0,56^{*}$ & $<0,0001^{*}$ & $-0,24^{*}$ & $0,02^{*}$ \\
\hline & Idade & $-0,32^{*}$ & $0,02^{*}$ & $0,32^{*}$ & $0,02^{*}$ & - & - \\
\hline & Hemoglobina & - & - & $-0,007$ & 0,96 & - & - \\
\hline \multirow{4}{*}{$\begin{array}{l}\text { Total } \\
\text { Mascu- } \\
\text { lino }\end{array}$} & Creatinina & $-0,22$ & 0,0008 & - & - & 0,24 & 0,005 \\
\hline & TFG & 0,33 & $<0,0001$ & 0,43 & $<0,0001$ & $-0,65$ & $<0,0001$ \\
\hline & Idade & $-0,37$ & $<0,0001$ & $-0,14$ & 0,08 & - & - \\
\hline & Hemoglobina & - & - & 0,19 & 0,03 & - & - \\
\hline
\end{tabular}

${ }^{*} p<0,05$, estatisticamente significativo; $r>0,3$ correlação positiva; TFG: Taxa de Filtração Glomerular; IMC: Índice de massa corpórea.

Quanto à variável idade observou-se que houve correlação significativa com os valores de creatinina $(r=0,47$ - regular, $\mathrm{p}=0,02$ ) nos pacientes do sexo feminino do grupo teste e com a TFG $(r=0,6$ - regular, $p<0,0001)$ nos pacientes do sexo masculino do grupo teste.

\section{Discussão}

A dosagem de creatinina sérica foi utilizada como parâmetro para selecionar os pacientes que apresentaram quadro sugestivo de doença renal, a partir da equação de CockcroftGault que calcula a TGF'. A TFG além de classificar o grau de lesão dos rins também é um marcador sensível do número de néfrons funcionais sendo uma ferramenta importante para as alterações na função renal. No entanto, a dosagem de creatinina sérica isolada não é suficiente para a confirmação diagnóstica da DRC, pois tem uma baixa sensibilidade nos primeiros estágios da doença, apresentando alteração somente 
quando 50 a $70 \%$ da atividade renal está comprometida 5 .

Em um estudo sobre a prevalência de anemia e insuficiência renal em portadores de insuficiência cardíaca nãohospitalizados ${ }^{16}$, foi observado que se a análise fosse feita a partir da dosagem isolada de creatinina sérica, com o critério de $1,3 \mathrm{mg} / \mathrm{dL}$, apenas $18,3 \%$ da amostra teria DRC, enquanto que na realidade o percentual foi de $74 \%$. O recomendado é o estudo da depuração de creatinina plasmática, porém, torna-se inviável devido à complexidade do procedimento e aos erros cometidos durante a coleta de urina de 24 horas, contribuindo então para que a realização da TFG estimada seja mais executada na rotina laboratorial ${ }^{11}$.

No presente trabalho uma porcentagem de 78,2\% (86/110) dos pacientes do grupo teste era do sexo masculino, assim como foi constatado em uma pesquisa, que encontraram $68,8 \%$ e $58 \%$ de pacientes do sexo masculino, respectivamente, porém estes estavam em tratamento hemodialítico ${ }^{14,17}$. Essa proporção pode ser explicada pelo maior cuidado que a mulher tem com a própria saúde, tanto em relação à realização de medidas preventivas, quanto em relação ao tratamento adequado para doenças crônicas como DM e HA, retardando o aparecimento de outras doenças como a DRC. Segundo uma pesquisa realizada pela Sociedade Brasileira de Urologia (SBU), mesmo com grande acesso à informação os homens ainda negligenciam a própria saúde ${ }^{15}$.

A anemia esteve presente em $31,8 \%$ dos pacientes com doença renal, e destes, 65,7\% (23/35) eram do sexo masculino. A análise estatística apresentou correlação positiva entre hemoglobina baixa com o aumento da idade e a alteração da TFG nos pacientes masculinos do grupo teste, sendo que a média de idade foi acima de 60 anos. $O$ envelhecimento dos indivíduos do sexo masculino leva a uma diminuição fisiológica dos níveis séricos de andrógenos que afeta sensivelmente o tecido eritroblástico à ação da eritropoietina, contribuindo assim, para a diminuição da eritropoiese, reduzindo consequentemente os valores de hemoglobina decorrente da produção prejudicada 5 . No sexo feminino do grupo teste, 34,3\% (12/35) apresentaram anemia e houve correlação positiva entre hemoglobina baixa e idade.

Em outro estudo foi verificado que a anemia está relacionada com a DRC independente do estágio, sendo que 39\% dos pacientes no seu estudo foram classificados no estágio 5 da $\mathrm{DRC}$, tendo em vista que eram pacientes em fase pré-dialítica ${ }^{18}$. O presente estudo também apresentou pacientes com anemia em vários estágios da $\mathrm{DRC}$, sendo mais prevalente no estágio 3 , cerca de $34,3 \%$ (23/67) dos pacientes.

A investigação da anemia é necessária em todo paciente que apresentar depuração de creatinina estimada menor que
$60 \mathrm{~mL} / \mathrm{min} / 1,73 \mathrm{~m}^{2}$, visto que a anemia é uma complicação frequentemente observada em pacientes portadores de DRC e está relacionada com o grau da DRC, corroborando com outra pesquisa ${ }^{19}$.

Em uma pesquisa foi descrito que a anemia está associada à piora da qualidade de vida e ao aumento da morbimortalidade na população com DRC, além de contribuir para a progressão da $D_{R C}{ }^{6,20}$. Acredita-se que a perda de massa renal seja a principal causa da anemia na DRC devido à diminuição da produção de eritropoietina que é um hormônio produzido por fibroblastos renais, estimuladores da produção dos eritrócitos na medula óssea, e tem uma ação inibitória na via das caspases que age como antiapoptóticos nas células precursoras das células vermelhas na medula óssea. Além disso, pode haver deficiência de eritropoietina por deficiência de ferro, folato e vitamina B12, perda sanguínea, hemólise ou inflamação ${ }^{6}$.

Recomenda-se a realização de hemograma, saturação de transferrina e ferritina sérica para pacientes com DRC, visto que a anemia na DRC é normocítica e normocrômica, porém pode haver deficiência de ferro devido ao processo inflamatório a que este paciente se encontra que pode alterar as características hematimétricas da anemia ${ }^{21}$.

No grupo teste observou-se que dentre os 110 pacientes, $91,8 \%(101 / 110)$ apresentavam idade acima de 60 anos indicando que a correlação entre a variável idade e a DRC é significativa. Assim, é notável que a idade é considerada um fator de risco da DRC, visto que, ocorre uma diminuição fisiológica da filtração glomerular em pacientes com idade mais avançada'. A lesão renal em decorrência da idade avançada é devido à perda do número de néfrons, e após os 40 anos de idade, a TFG diminui $4,6 \mathrm{ml} / \mathrm{min} / 1,73 \mathrm{~m}^{2}$ em homens e $7,1 \mathrm{ml} / \mathrm{min} / 1,73 \mathrm{~m}^{2}$ em mulheres a cada década refletindo na redução acentuada do fluxo sanguíneo renal e consequentemente da filtração glomerular (FG) ${ }^{22}$. Aos 80 anos a FG diminui em 40 a 50\% nos indivíduos sadios. Em 2002, Jones encontrou maior prevalência de perda renal em pacientes idosos ${ }^{23}$, corroborando com os resultados desse presente estudo que apresentou correlação significativamente positiva entre a idade e os níveis de creatinina para o sexo feminino e a TFG para o sexo masculino.

A obesidade vem sendo considerada outro fator de risco para o desenvolvimento de lesão glomerular e progressão para a falência funcional independentemente da ocorrência simultânea de HA e DM²4. As alterações glomerulares na obesidade caracterizam-se por hiperfluxo e hipertensão glomerular que evoluem para glomeruloesclerose e proteinúria acompanhada por rápida perda da função renal ${ }^{5}$. Além disso, a gordura pode comprimir os rins e lesionar a medula renal decorrente da deposição da matriz extracelular e assim influenciar no 
aumento da pressão intersticial e consequentemente maior reabsorção de sódio e $H A^{24,26}$.

Em um estudo observou-se que a obesidade pode causar comprometimento renal de progressão lenta, e em torno de $10 \%$ dos pacientes podem evoluir para $\mathrm{DRC}^{27}$. Além disso, acreditam que o dano renal relacionado à obesidade seria consequente de uma glomerulopatia e não decorrente de uma HA e DM. Tozowa ${ }^{28}$ notaram que a obesidade aumentava em $54 \%$ o risco de lesão renal sem relação nenhuma com outros fatores como HA, DM, nefropatia e tabagismo.

No presente estudo observa-se uma correlação entre o IMC e a TFG nos pacientes femininos do grupo teste, considerando que $58,3 \%$ (14/24) estavam acima do peso. No sexo masculino, a correlação entre IMC e TFG foi positiva, sendo que $75 \%$ (60/86) estavam acima do peso. O IMC também teve correlação significativamente positiva com hemoglobina e idade nos pacientes masculinos do grupo teste. Dessa maneira, o valor de IMC acima de $25 \mathrm{Kg} / \mathrm{m}^{2}$, adotado pela OMS ${ }^{18}$ para classificar sobrepeso ou obesidade, foi utilizado como critério para estimar esse quadro. Assim, foi observado nos estágios 3 e 4 da DRC uma prevalência significativa de obesos e sobrepeso, na qual, corresponde à $62,7 \%(42 / 67)$ e $54,5 \%$ (12/22) respectivamente, independente do sexo. Já em outro estudo, a presença de sobrepeso e obesidade foi consideravelmente maior no estágio 2 (77\%) quando comparado ao estágio 4 (50\%) e estágio 5 (38\%), visto que o IMC foi menor nos estágios mais avançados em relação ao estágio $2^{18}$.

\section{Conclusão}

A partir da análise dos resultados foi possível concluir que em ambos os grupos houve correlação entre as variáveis e a DRC. No entanto, foi possível observar que as variáveis associadas à DRC, como idade, IMC, hemoglobina, creatinina, TFG foram relevantes para o desenvolvimento dessa condição clínica. A idade, a anemia e a TFG apresentaram as maiores correlações no grupo teste. É importante ressaltar que os pacientes desse estudo são ambulatoriais e estão em estágios menos avançado da DRC e para uma análise mais detalhada seria necessário à realização de outros exames laboratoriais.

\section{Referências Bibliográficas}

1. Bastos MG, Bregman R, Kirsztajin GM. Doença renal crônica: frequente e grave, mas também prevenível e tratável. Rev Assoc Med Bras. 2010;56(2):248-53.

2. Diretrizes Brasileiras de Doença Renal Crônica. J Bras Nefrol. 2004;26(Supl1):S1-S49.

3. Bastos MG, Kirsztajin GM. Doença renal crônica: importância do diagnóstico precoce, encaminhamento imediato e aborda- gem interdisciplinar estruturada para melhora do desfecho em pacientes ainda não submetidos à diálise. J. Bras. Nefrol. 201;33(1):93-108.

4. Shemesh O, Golbetz H, Kriss JP, Myers BD. Limitations of creatinine as a filtration marker in glomerulopathic patients. Kidney Int. 1985;28(5):830-8.

5. Fox SI. Fisiologia Humana. 7. Ed. Barueri: Manole; 2007.

6. Abensur H, Bastos MG, Canziani MEF. Aspectos atuais da anemia na doença renal crônica; Actual aspects of anemia in chronic kidney disease. J Bras Nefrol. 2006;28(2):104-7.

7. Alvarez-Hernández X, Licéaga J, McKay IC, Brock JH. Induction of hypoferremia and modulation of macrophage iron metabolism by tumor necrosis factor. Lab Invest. 1989;61(3):319-22.

8. Abensur H. Biomarcadores na Nefrologia. Sociedade Brasileira de Nefrologia. 2011.

9. Lemos ADR, Ismael LAS, Boato CCM, Borges MTF, Rondó PHDC. A hepcidina como parâmetro bioquímico na avaliação da anemia por deficiência de ferro. Rev Assoc Med Bras. 2010;56(5):596-9.

10. Deicher R, Horl WH. New insights into the regulation of iron homeostasis. European journal of clinical investigation. 2006;36(5):301-9.

11. Sodré FL, Costa JCB, Lima JCC. Avaliação da função e da lesão renal: um desafio laboratorial. J bras patol med lab. 2007;43(5):329-37.

12. Brasil. Ministério da Saúde (2014). Secretaria de Atenção à Saúde. Departamento de Atenção Especializada e Temática. Diretrizes Clínicas para o Cuidado ao paciente com Doença Renal Crônica - DRC no Sistema Único de Saúde/ Ministério da Saúde. Secretaria de Atenção à Saúde. Departamento de Atenção Especializada e Temática. - Brasília: Ministério da Saúde, 1-37.

13. Bastos MG, Abrita RR, Almeida EC, Maria D, Costa N, Gonçalves JDA et al. Doença Renal Crônica: Problemas e Soluções. J Bras Nefrol. 2004;26(4):202-15.

14. Bueno CS, Frizzo MN. Anemia in chronic kidney disease in a Hospital in the Northwest region to the State of Rio Grande do Sul. J Bras Nefrol. 2014;36(3):304-14.

15. Sociedade Brasileira de Urologia. Saúde do Homem. 2014. Disponível em: <http://www.sbu.org.br/publico/pdf/pesquisasaude-do-homem-2014.pdf>. Acesso em 3 jun. 2015.

16. Reis FJFB, Fernandes MAS, Bitencourt AGV, Neves FBCS, Kuwano AT, França VHP, et al. Prevalência de anemia e insuficiência renal em portadores de insuficiência cardíaca nãohospitalizados. Arq. Bras. Cardiol. 2009;93(3):268-74.

17. Sesso RC, Lopes AA, Thomé FS, Lugon JR, Watanabe Y, Santos DR. Report of the brazilian chronic dialysis census 2012. J Bras Nefrol. 2014;36(1):48-53. 
18. Canziani MEF, Bastos MG, Bregman R, Pecoits Filho R, Tomyiama C, Draibe SA, et al. Deficiência de ferro e anemia na doença renal crônica. J Bras Nefrol. 2006;28(2):86-90.

19. Abensur H. Anemia da doença renal crônica. J Bras Nefrol. 2004;26(3):26-8.

20. Beusterien KM, Nissenson AR, Port FK, Kelly M, Steinwald B, Ware JR. The effects of recombinant human erythropoietin on functional health and well-being in chronic dialysis patients. J Am Soc Nephrol. 1996;7(5):763-73.

21. Ribeiro-Alves MA, Gordan PA. Diagnosis of anemia in patients with chronic kidney disease. J Bras Nefrol. 2014;36(1):9-12.

22. Cohney S, Kanellis J, Howell M. Donor renal function. Nephrology. 2010;15(s1):S137-S145.

23. Jones CA, Francis ME, Eberhardt MS, Chavers B, Coresh J, Engelgau $M$, et al. Microalbuminuria in the US population: third national health and nutrition examination survey. Am J Kidney Dis. 2002;39(3):445-59.

24. Paula RB, Fernandes N, Carmo VMP, Andrade LCF, Bastos MG. Obesidade e doença renal crônica. J Bras Nefrol. 2006;28(3):158-64.

25. Hall JE, Crook ED, Jones DW, Wofford MR, Dubbert PM. Mechanisms of obesity-associated cardiovascular and renal disease. A J Med Sci. 2002;324(3):127-37.

26. Henegar JR, Bigler SA, Henegar LK, Tyagi SC, Hall JE. Functional and structural changes in the kidney in the early stages of obesity. J Am Soc Nephrol. 2001;12(6):1211-7.

27. Kambham N, Markowitz GS, Valeri AM, Lin J, D'Agati VD. Obesity-related glomerulopathy: an emerging epidemic. Kidney Int. 2001;59(4):1498-509.

28. Tozawa M, Iseki K, Iseki C, Oshiro S, Ikemiya Y, Takishit S. Influence of smoking and obesity on the development of proteinuria. Kidney Int. 2002;62(3):956-62. 